\title{
The object-superiority effect by threshold assessment
}

\author{
DEAN G. PURCELL \\ Oakland University, Rochester, Michigan \\ ALAN L. STEWART \\ Stevens Institute of Technology, Hoboken, New Jersey \\ and \\ ANGELA M. GIACOLETTI \\ Oakland University, Rochester, Michigan
}

\begin{abstract}
Targets embedded within three-dimensional stimuli had lower identification thresholds than did targets embedded within two-dimensional stimuli. This finding extends the object-superiority effect to include duration thresholds for identification. The pattern of identification errors supports previous findings that structurally relevant targets are harder to identify than nonstructurally relevant targets.
\end{abstract}

Weisstein and her colleagues used tachistoscopically presented line drawings in their studies of the objectsuperiority effect (OSE). They found that target lines were identified best when they were part of a pattern that was seen as a three-dimensional figure. The same target was identified less well if it was part of a stimulus pattern that was seen as a two-dimensional figure (Lanze, Weisstein, \& Harris, 1982; Weisstein \& Harris, 1974; Weisstein, Williams, \& Harris, 1982).

The OSE is indicated whenever targets contained within three-dimensional contexts are recognized more quickly than the same targets contained within two-dimensional contexts. Differences in performance on individual target lines have seldom been reported (see, e.g., Enns \& Gilani, 1988; Enns \& Prinzmetal, 1984; Klein, 1978; McClelland, 1978; Weisstein \& Harris, 1974; Weisstein et al., 1982; Williams \& Weisstein, 1978; Williams \& Weisstein, 1984), although there have been two exceptions to this trend (McClelland, 1978; McClelland \& Miller, 1979). Performance data on individual target lines are important, because they permit analysis of factors such as structural relevance, as well as target line position relative to the interior-exterior of the stimulus.

We determined identification thresholds by using a fouralternative forced-choice procedure. We directly determined these thresholds for target lines in three- and twodimensional contexts. We also recorded error rates for each of the four target lines in each of the two contexts.

This research was supported by an Oakland University Faculty Research Grant. We thank Michael Botwin for writing the software used to control the experiment. The data were presented at the 28th annual meeting of the Psychonomic Society (Purcell, Stewart, \& Giacoletti, 1987). D. G. Purcell is in the Department of Psychology, Oakland University, Rochester, MI 48309-4401. A. L. Stewart is with the Human Factors Program, Department of Management and Engineering Management, Stevens Institute of Technology, Hoboken, NJ 07030.
Weisstein and her colleagues argued that the OSE results from a perceptual advantage for three-dimensional stimuli. They used percent correct line identification as the dependent variable. We hypothesized that the same stimuli should also produce the OSE when the line identification threshold was the dependent variable. Yet although this prediction appears straightforward, minor changes in the experimental procedures can reverse the OSE.

Widmayer and Purcell (1982) found that search time was longer for target lines in a three-dimensional context as opposed to a two-dimensional context. Pomerantz (1981, p. 178) also found that the counting of diagonal lines was slower in a three-dimensional context than in a two-dimensional context. Similarly, Klein (1978) found that the reaction time to identify the slope of the target line was longer for lines embedded within a context that looked like a three-dimensional object. Thus, in tasks in which time to respond is the dependent variable, a threedimensional context does not lead to superior performance, even if the stimuli are the same as those used by Weisstein and Harris (1974). Consequently, although our prediction was straightforward, the findings were not guaranteed (Purcell \& Stewart, 1991).

\section{METHOD}

We will hold to the following conventions. The line to be identified will be called the target line. The visual context will be all lines other than the target line. The three- and two-dimensional stimuli will refer to the target lines combined with a visual context.

\begin{abstract}
Subjects
Twenty-seven naive undergraduates from introductory psychology classes volunteered as subjects. All had normal or corrected-to-normal vision. Two subjects were eliminated from the experiment because their error rates were either too high or too low to allow correct threshold assessment. Consequently, the data for 25 subjects are presented.
\end{abstract}




\section{Apparatus and Stimuli}

Eight stimuli were created. These are depicted in Figure 1. They are based on the three- and two-dimensional stimuli used by Williams and Weisstein (1984). The location of the fixation point appears as part of each stimulus shown in Figure 1. During the experiment, the fixation point was not presented with the target. The fixation field disappeared when the stimulus came on. The fixation dot was about $0.07^{\circ}$ in diameter.

The three-dimensional context was $1.61^{\circ}$ square. The two-dimensional context had the same height as did the three-dimensional context, but it was $1.09^{\circ}$ wide. The line segments forming the stimuli had a strip width of $0.07^{\circ}$. The target and fixation fields were $9^{\circ}$ wide by $6^{\circ}$ high. The positions of the four target lines, about the fixation point, were the same for each context. The near end of the target line was $0.66^{\circ}$ from fixation, and the far end was $0.87^{\circ}$ from fixation.

The target luminance was adjusted for each subject during practice trials $\left(27.41 \mathrm{~cd} / \mathrm{m}^{2}\right.$ [3 subjects]), $10.28 \mathrm{~cd} / \mathrm{m}^{2}$ [12 subjects], $5.99 \mathrm{~cd} / \mathrm{m}^{2}$ [4 subjects], $4.28 \mathrm{~cd} / \mathrm{m}^{2}$ [6 subjects]). The physical contrast of the stimulus was not changed by this procedure. However, luminance summation from the fixation field would reduce the effective stimulus contrast as target field luminance was lowered (Eriksen, 1966).

The fixation field remained constant at $21 \mathrm{~cd} / \mathrm{m}^{2}$. The equipment was programmed to present the fixation field for $750 \mathrm{msec}$. This field was replaced by the target field for a duration determined by the PEST threshold assessment procedure. The fixation field then returned for $750 \mathrm{msec}$. The experiment was run in a black room illuminated with overhead fluorescent lights. The walls reflected approximately $1.7 \mathrm{~cd} / \mathrm{m}^{2}$.

\section{Procedure}

Duration thresholds for a criterion level of target identification were determined with the Best PEST algorithm (Lieberman \& Pentland, 1982). For a four-alternative forced-choice response, this algorithm sets the threshold at the duration where the subject correctly locates or identifies the target on $62.5 \%$ of the trials. If the PEST procedure works properly, there should be $37.5 \%$ errors in each condition.

As can be seen in Figure 1, the target could appear in one of four positions within each dimensional context. Using the fixation point as a reference, the target line could appear above left, above right, below left, or below right. The subject pushed a joystick toward the quadrant in which the target line was judged to have been presented.

Because a forced-choice procedure was used, the subjects were instructed to respond after each stimulus presentation, even if they had to guess. On each trial, the type of context (three- or two-dimensional) was chosen at random, as was the target line. A constraint was applied to the choice. Each of the four target lines appeared equally often within each of the two contexts, and the contexts were presented equal numbers of times.

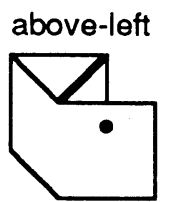

$50.40 \pm 8.29$

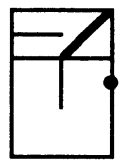

$57.60 \pm 8.36$

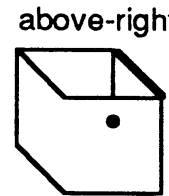

$48.40 \pm 7.69$

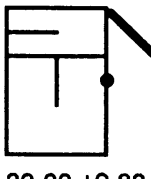

$30.00 \pm 9.83$

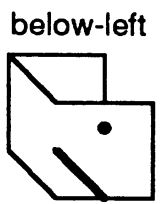

$27.20 \pm 8.64$

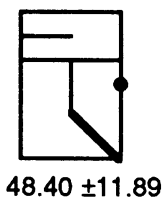

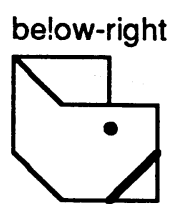

$16.00 \pm 6.08$

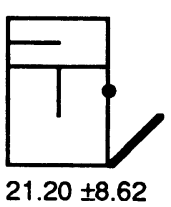

Figure 1. Targets and contexts used in Experiment 1. Percent error and $95 \%$ confidence intervals are given under each figure. For the sake of clarity, target lines in this figure are rendered in bold. In the experiment, target lines had the same thickness as the context lines. Fixation point location is depicted for reference only. During the experiment, the fixation point terminated at target onset.
The subjects were instructed to attend to the target line and to ignore its context. They began each trial by pressing a start button. After the stimulus presentation, the subject responded with a joystick movement, and then a buttonpress to record that movement. Immediately following each response, a computer-generated tone indicated whether the response was correct or incorrect. The experimenter monitored the experiment, changed the stimulus cards, and keyed the onset of a buzzing sound, which signaled to the subject that he/she was free to begin the next trial.

The procedures were explained to each subject during a practice session. Before practice, the subjects were shown each of the eight combinations of context and target lines outside the tachistoscope. Each subject was then given a block of 16 practice trials, with the target duration set at $60 \mathrm{msec}$. This practice block had two presentations of each of the eight stimuli. If subjects had any difficulty with the task, the target field luminance was increased, and the practice session was rerun. The training was repeated until no errors were made by the subject.

The subjects were tested individually for $.5 \mathrm{~h}$. The duration threshold was the stimulus duration of the last trial in the 40-trial thresholdsetting sequence. Because there were two conditions, the subjects received a total of 80 experimental trials. Each of the four target-line positions was presented 10 times within each of the two contexts.

\section{RESULTS}

Consistent with previous work on the OSE, the identification threshold for target lines contained within the three-dimensional context $(24.4 \mathrm{msec})$ was lower than that for targets contained within the two-dimensional context $(32.3 \mathrm{msec})$. A repeated measures analysis of variance (ANOVA) showed that this 7.88-msec difference was statistically reliable $\left[F(1,24)=8.8, p<.01, \eta^{2}=.27\right]$.

Separate analyses were done on errors to determine whether error rates differed from the $37.5 \%$ predicted by the optimum performance of the PEST procedure. The average error rate for the three-dimensional condition was $35.5 \%$. This value was not reliably different from the expected value, although it approached statistical reliability $\left[t(24)=-1.80, p=.08, r^{2}=.12\right]$. The direction of this result suggests that the threshold for the three-dimensional condition was set too high. If the threshold for the threedimensional condition was set too high, the OSE would be underestimated. For the two-dimensional condition, there were $39.3 \%$ errors. This also was not reliably different from the expected error rate $[t(24)=0.18]$.

A further analysis was made of the errors at each of the four line positions for the two levels of dimensionality. Our assumption was that each target-line position would contribute equally to the threshold estimate. Errors should be equivalent for all target-line positions under both conditions of dimensionality. Errors for these eight conditions produced a complex pattern. Figure 1 includes the mean error rate and the $95 \%$ confidence interval for each condition of the experiment.

A repeated measures ANOVA was run on the error data for each of the four target lines at each of the two levels of dimensionality. The main effect of stimulus dimensionality was not statistically reliable $[F(1,24)=2.95, p=.1$, $\left.\eta^{2}=.11\right]$. The main effect of vertical position (above vs. below fixation) was statistically reliable $[F(1,24)=37.37$, $\left.p<.001, \eta^{2}=.61\right]$. The main effect of horizontal position (left vs. right of fixation) was also statistically reli- 
able $\left[F(1,24)=16.32, p<.001, \eta^{2}=.40\right]$. Dimensionality interacted with both horizontal $[F(1,24)=10.35, p<$ $\left..005, \eta^{2}=.30\right]$ and vertical position $[F(1,24)=14.27$, $\left.p=.001, \eta^{2}=.37\right]$. The two-way interaction of vertical position $\times$ horizontal position was not reliable $[F(1,24)=$ 0.82 ], nor was the three-way interaction of dimension $x$ vertical position $\times$ horizontal position $[F(1,24)=0.5]$.

To clarify the relationship between relevant and nonrelevant target lines, an ANOVA was run on the percent error data for the three-dimensional stimuli. With our threedimensional stimuli, vertical position could be construed as a manipulation of target-line relevance; viewed thus, upper (relevant) targets produced $27.8 \%$ more errors than did lower (nonrelevant) targets. This main effect of vertical position was reliable $[F(1,24)=40.48, p<.001$, $\left.\eta^{2}=.63\right]$. The effect of horizontal position approached statistical reliability $\left[F(1,24)=3.19, p<.1, \eta^{2}=.12\right]$. The interaction of these two variables was not statistically reliable $\left[F(1,24)=1.14, p>.05, \eta^{2}=.05\right]$.

The threshold data showed that the OSE occurs when the target-line threshold is assessed directly. The error data show that, within a given dimension, line placement affects identification. This shows that factors other than stimulus dimensionality contribute to performance on this task.

\section{DISCUSSION}

\section{Confounds and Controls}

A simple bias to respond to the right would account for most of the effect of horizontal position. This explanation is challenged by the interaction of dimensionality with horizontal position. This interaction reflects a greater effect of horizontal position for the two-dimensional stimuli than for the three-dimensional stimuli. The greater horizontal position effects for two-dimensional contexts are probably due to the fact that the target lines on the right of fixation are relatively isolated from the context. They are not contained within the two-dimensional figure as the target lines on the left are, so they should be easier to extract. This result, with our two-dimensional stimuli, is consistent with the results from McClelland and Miller (1979, Experiment 1).

The effect of vertical position can be explained by a simple bias to respond down. However, the interaction of dimensionality with vertical position shows larger effects of vertical position for three-dimensional than for two-dimensional stimuli. The separate analysis of errors in the three-dimensional condition shows that vertical position (i.e., target relevance) has a strong effect on target location thresholds. Targets below the fixation point are less structurally relevant than those above the fixation point. Hence, the effect of vertical position may reflect an effect of structural relevance.

In the present experiment, targets structurally relevant to the threedimensional stimulus produce the greatest number of errors (see Figure 1). This result is consistent with Lanze et al. (1982, Experiment 3), who found that their least structurally relevant target lines in threedimensional contexts produced the best performance. These results are not consistent with those of McClelland and Miller (1979, Experiment 2), who found that the greater the structural relevance of a target line, the better it was identified in a three-dimensional stimulus.

As McClelland and Miller (1979) have pointed out, the context in their Experiment 2 interacted with the target lines to change the dimensionality of the stimulus. The context with the structurally relevant target lines appeared to be more three-dimensional than the context with the nonstructurally relevant target lines. Consequently, the effect of structural relevance that McClelland and Miller reported having found in their Experiment 2 could have been due to stimulus dimensionality.

In their Experiment 3, McClelland and Miller (1979) controlled for dimensionality by incorporating both relevant and nonrelevant target stimuli into objects that appeared to be three-dimensional. Yet although this procedure controlled for the confounding of target relevance with dimensionality, it introduced another confound.

McClelland and Miller's (1979, Experiment 3) relevant target lines produced three-dimensional stimuli (hurdles) whose two versions (upright vs. knocked over) differed greatly in external outline. With the hurdle stimuli, the target line was redundant with the version of the hurdle presented. When the target lines were not relevant, the outlines of the three-dimensional stimuli (objects) were identical. Given these differences between object and hurdle stimuli, subjects could use different strategies to identify target lines associated with them.

With the relevant target lines, subjects need only discriminate between the upright and knocked over versions of the hurdle stimuli to perform the task. With the nonrelevant target lines, subjects would have to search the interior of identical object contexts. Since the outlines of the object contexts were not redundant with the target line, performance with the nonrelevant targets should suffer (Enns \& Prinzmetal, 1984). See Weisstein et al. (1982) for a discussion of other confounding factors contributing to superior performance on relevant targets contained within the hurdle stimuli of McClelland and Miller (1979).

We found that identification performance was poorer with relevant target lines than with nonrelevant target lines, as did Lanze et al. (1982). Except for the stimulus with the target line in the above-right position, our three-dimensional stimuli had the same outline. With the possible exception of the above-right condition, our subjects could not have been performing an object discrimination task on the basis of the stimulus outline. They must have been searching for the target-line location. Thus, our experiment was free from the confound of McClelland and Miller's Experiment 3.

Our three-dimensional stimulus error rates and those of Lanze et al. (1982) are lower for nonrelevant targets than for relevant targets. This may be because the nonrelevant targets can be extracted or isolated from the context more easily than can relevant targets (Mermelstein, Banks, \& Prinzmetal, 1979; Pomerantz, 1981; Prinzmetal \& Banks, 1977).

\section{Conclusion}

Our results are clear; we have extended the conditions under which the OSE may be found to those of direct assessment of target-line threshold. The structural relevance of the target line, and the dimensionality of its context, can affect target perception. The effect of context on the target line, although interesting in its own right, may serve to obscure the basic problem of interest in studies of the OSE. This problem concerns whether some configurations of visual stimuli are perceptually advantaged over others.

The determination of identification thresholds for specific target lines allows study of the joint effects of dimensionality and structural relevance. However, the problem of object perceptual superiority may be studied more directly by using a technique that asks the subject simply to detect the presence of the stimulus. This technique has been used to study the face-detection effect (Gorea \& Julesz, 1990; Purcell \& Stewart, $1981,1986,1988$ ), and what we call the object-detection effect (Purcell, Stewart, \& Giacoletti, 1987; Purcell \& Stewart, 1991). This technique has also been used to study the detectability of words (Doyle \& Leach, 1988).

\section{REFERENCES}

DOYLE, J. R., \& LEACH, C. (1988). Word superiority in signal detection: Barely a glimpse, yet reading nonetheless. Cognitive Psychology, 20, 283-318.

EnNS, J. T., \& Gilani, A. B. (1988). Three-dimensionality and discriminability in the object-superiority effect. Perception \& Psychophysics, 44, 243-256.

EnNS, J. T., \& Prinzmetal, W. (1984). The role of redundancy in the object-line effect. Perception \& Psychophysics, 35, 22-32.

ERIKSEN, C. W. (1966). Temporal luminance summation effects in backward and forward masking. Perception \& Psychophysics, 1, 87-92.

GoreA, A., \& Julesz, B. (1990). Context superiority in a detection task with line-element stimuli: A low-level effect. Perception, 19, 5-16.

KLEIN, R. (1978). Visual detection of line segments: Two exceptions to the object superiority effect. Perception \& Psychophysics, 24, 237-242. 
Lanze, M., Weisstein, N., \& Harris, J. R. (1982). Perceived depth vs. structural relevance in the object-superiority effect. Perception \& Psychophysics, 31, 376-382.

Lieberman, H. R., \& Pentland, A. P. (1982). Microcomputer-based estimation of psychophysical thresholds: The Best PEST. Behavior Research Methods \& Instrumentation, 14, 21-25.

MCClelland, J. L. (1978). Perception and masking of wholes and parts. Journal of Experimental Psychology: Human Perception \& Performance, 4, 210-223.

McClelland, J. L., \& Miller, J. (1979). Structural factors in figure perception. Perception \& Psychophysics, 26, 221-229.

Mermelstein, R., Banks, W., \& Prinzmetal, W. (1979). Figural goodness effects in perception and memory. Perception \& Psychophysics, 26, 472-480.

Pomerantz, J. R. (1981). Perceptual organization. In M. Kubovoy \& J. R. Pomerantz (Eds.), Perceptual organization (pp. 141-180). Hillsdale, NJ: Erlbaum.

Prinzmetal, W., \& Banks, W. P. (1977). Good continuation affects visual detection. Perception \& Psychophysics, 21, 389-395.

Purcell, D. G., \& Stewart, A. L. (1981). A face superiority effect. Bulletin of the Psychonomic Society, 18, 73. (Abstract)

Purcell, D. G., \& Stewart, A. L. (1986). The face-detection effect. Bulletin of the Psychonomic Society, 24, 118-120.

Purcell, D. G., \& STEWART, A. L. (1988). The face-detection effect: Configuration enhances detection. Perception \& Psychophysics, 43, 355-366.
Purcell, D. G., \& Stewart, A. L. (1991). The object-detection effect: Configuration enhances perception. Perception \& Psychophysics, 50, 215-224.

Purcell, D. G., Stewart, A. L., \& Giacoletti, A. M. (1987). The object detection effect. Bulletin of the Psychonomic Society, 25, 347. (Abstract)

Weisstein, N., \& Harris, C. S. (1974). Visual detection of line segments: An object-superiority effect. Science, 186, 752-755.

Weisstein, N., Williams, M. C., \& HarRIs, C. S. (1982). Depth, connectedness, and structural relevance in the object-superiority effect: Line segments are harder to see in flatter patterns. Perception, 11, 5-17.

Widmayer, M., \& PURCEll, D. G. (1982). Visual scanning of line segments: Object superiority and its reversal. Bulletin of the Psychonomic Society, 19, 353-354.

Williams, A., \& Weisstein, N. (1978). Line segments are perceived better in a coherent context than alone: An object-line effect in visual perception. Memory \& Cognition, 6, 85-90.

Williams, M. C., \& Weisstein, N. (1984). The effect of perceived depth and connectedness on metacontrast functions. Vision Research, 24, 1279-1288.

(Manuscript received April 3, 1993.) 